\author{
Military Technical College \\ Kobry Elkobbah, \\ Cairo, Egypt
}

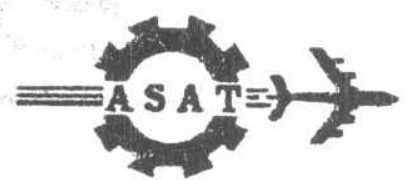

$8^{\text {th }}$ International Conference on Aerospace Sciences \& Aviation Technology

\title{
MODIFIED METHOD FOR SIGNAL TO NOISE RATIO IMPROVEMENT USING DIGITAL FILTERS
}

\author{
M.I.M.Dessouky * , M.M.Hadhoud ${ }^{* *}$, F. E. Abd El-Samie ${ }^{\star * *}$
}

\begin{abstract}
Traditionally, digital filters were used for signal-to-noise ratio (SNR) improvement. This paper proposes a modified method to enhance and improve the digital filter performance. This method is based on the combination of coherent time averaging technique and digital filtering. This paper discusses the processing of single and multiple input sinusoidal signals with additive noise. By using this method in processing multiple signals, we can isolate the weak in-band signals from the strong out-of-band signals, which consequently improve the weak signal delectability. Cascaded digital filtering is applied using similar or different types of digital filters. A comparison study is provided for single stage and cascaded stages digital filters. It is shown that this modified method leads to better results in SNR improvement of weak signals than in the case of the direct application of digital filtering.
\end{abstract}

\section{INTRODUCTION}

The main goal of digital signal processing (DSP) is to estimate the characteristic parameters of a signal or to transform the signal into a form that is more desirable to allow the detection of the signal in an easy method [1]. In this paper we are going to use the linear spectral estimation technique (FFT) [1] as a spectral estimation method. To improve the SNR, different techniques will be examined. These techniques are the coherent time averaging [2] and filtering techniques. In addition, we also introduce digital filtering of preprocessed signals using coherent time averaging to isolate weak signals from strong signals.

\section{INPUT SIGNALS}

For a received signal comprising more than one sinusoidal component with different frequencies, different phase and different amplitudes, the mathematical representation is given by [2]

$$
x(k)=\sum_{i=1}^{N_{S}}\left[x_{i}(k)+n(k)\right]=\sum_{i=1}^{N_{S}}\left[A_{i} \sin \left(\omega_{i} k T+\phi_{i}\right)+n(k)\right]
$$

\footnotetext{
*Assoc. prof., Dept. of Elec. Eng. Menoufia university, Menouf, Egypt. *AAssoc. prof., Dept. of Elec. Eng. Menoufia university, Menouf, Egypt. ${ }^{\star * \star}$ Graduate student, Dept. of Elec. Eng. Menoufia university, Menouf, Egypt.
} 
where:

$\mathrm{N}_{\mathbf{s}}$ is the number of sinusoidal signals merged together.

$A_{i}$ is the amplitudie of the $i^{\text {th }}$ signal and is responsible for determining its power.

$f_{i}$ is the frequency of the signal $i^{\text {th }}$ signal.

$\phi$, denotes the pinase of the $i^{\text {th }}$ signal.

$T$ is the samp'ing time.

In this paper, we choose the Fast Fourier Transform (FFT) as a method of measuring the spectral estimation peaks of the input signal. The discrete Fourier transform is given by the following equation [1]:

$$
X(m)=\sum_{k=0}^{N} x(k) \exp \left(-\frac{j 2 \pi m k}{N}\right)
$$

where

$x(k)$ : the input sequence of data.

$X(m)$ : the discrete Fourier transform of the input sequence .

$\mathrm{N}$ : the number of points .

$\mathrm{m}=0,1,2, \ldots \ldots \ldots \ldots \ldots, \mathrm{N}-1$

\section{COHERENT TIME AVERAGING}

In situations where a repetitive waveform is masked by random gaussian noise, crsherent time averaging can be most useful for signal enhancement. This technique consists of a summation of successive repetitions of a coherent signal in such a way that the signal reinforces itself, while the nois's, if it is random relative to the occurrences of the signal tends to cancel out. In this paper, we will concentrate on equal weight coherent time averaging $[3,4]$. This type of averager or smoother has a frequency response that has linear phase $(\theta)$ and a magnitude squared response given by the following, equation [5]:

$$
\left|H\left(e^{j \omega}\right)\right|^{2}=\left|\frac{\sin (M \theta)}{M \sin (\theta)}\right|^{2}
$$

where $\theta=(\omega T / 2)$

Thus, the SNR after summation of $M$ repetitions is given by [2]

$$
S N R_{M}=\sqrt{M} S N R_{1}
$$

where :

SNR $R_{M}$ : signal to noise ratio after $M$ times coherent time averaging.

$M$ : No. of averaging times.

SNR $_{1}$ : signal to noise ratio before coherent time averaging . 
the signal to noise ratio by a factor of $\sqrt{M}$.

\section{DIGITAL FILTERS}

Filtering is a process by which the frequency spectrum of a signal can be modified or reshaped according to some desired specifications. It may amplify or attenuate a range of frequency components. It separates two or more distinct signals that were purposely mixed in order to maximize channel utilization. Our concentration will be on recursive digital filters which is defined by the following equation [6]:

$y(n T)=\sum_{i=0}^{N} a_{i} x(n T-i T)-\sum_{i=1}^{N} b_{i} y(n T-i T)$

where $x(n T)$ is the input sequence and $y(n T)$ is the output sequence.

As in analog filters the approximation step in the design of digital filters is the process whereby a realizable transfer function satisfying prescribed specifications is obtained. Recursive filter approximations can be obtained from analog filter approximations using different methods [6]. In this paper, we will concentrate on the Bilinear Transformation method for designing the digital filters. This method has no aliasing errors due to applying the prewarping process before the bilinear transformation. Thus, the digital filter obtained by using the bilinear transformation method will have the same amplitude response as the analog filter at approximately the same frequency. The bilinear transformation steps can be summarized as discussed in [6].

This paper will study three types of approximations, which are Butterworth, Chebyshev and Inverse Chebyshev approximations. The detailed analysis for these approximations is given in $[6,7]$.

A cascade of digital recursive filters of $\mathrm{N}$ stages can be formed for satisfying certain specifications in loss characteristic. The types of approximations used to realize each stage may be similar or different.

\section{THE PROPOSED METHOD}

In this section, we are going to study the performance of the proposed method. The general block diagram for the entire different signal processing methods examined in this paper is depicted in Fig. (1). As illustrated in this figure, the output 1 gives the direct spectral estimation for the input signal using merely the periodogram technique [1]. The output 2 shows the output spectrum using digital filters only. The output 3 is the output result using the proposed method. This method is based on the application of coherent time averaging technique to the signal before digital filtering. This method can be applied with either single or multiple stage digital filters.

\section{PROCESSING RESULTS OF A SINGLE INPUT SIGNAL}

This section presents the results of the proposed technique. The method was implemented by software to perform the different required tasks which are the generation of the input signals (with different amplitudes, frequencies, phases and SNRs), simulation of the linear spectral estimation 
techniques using the $\mathrm{FFr}$ (periodogram) and simulation of the coherent time averaging technique. In addition, simulation design packages of single stage digital bandpass filters of different types (Butterworth, Chebyshev and Inverse Chebyshev) with different specifications and cascaded bandpass digital filter structures of similar and different types are elaborated.

In all of our study, we will select the frequencies of the input signals in the range from 0 to $25 \mathrm{kHz}$, a sampling frequency $\mathrm{f}_{\mathrm{s}}=50 \mathrm{kHz}$ and a number of points $N_{p}=512$ points. In general, the frequencies of the input signals can be chosen arbitrarily.

In this sfaction, we are going to examine the processing of a single signal in the presence of noise. Figures 2 to 4 show the spectrum of a single input sinusoidal signal with frequency $10 \mathrm{kHz}$ and phase $=0$ as the SNR decreases from 0 to $-10 \mathrm{~dB}$ in $5 \mathrm{~dB}$ steps. The figures illustrate that the peak of the input signal is completely immersed in noise and the detection of this input signal becomes very difficult as the SNR decreases to $-10 \mathrm{~dB}$.

Digital filters are used to improve the SNR and consequently the signal delsactability. Figures 5 to 7 depict the loss response of digital bandpass (Bistterworth, Chebyshev and Inverse Chebyshev) filters respectively with orders $n=2$ and 4 and with bandwidth extending from 8 to $12 \mathrm{kHz}$ (arbitrarily (chosen). Figures 8 to 10 give the output results of input signal with SNR $=-10$ dB filtered through the bandpass digital filters (Butterworth, Chebyshev and Inverse Chebyshev respectively) with order $n=4$. These results illustrate that no improvement is obtained in the in-band signal SNR and delectability due to the poor performance of the digital filters.

It is clear that there is a need for a proposed method to enhance the poor performance of the digital filters. The proposed method of combining the coherent time averaging with filtering techniques is intended to improve the weak signal delectability. The coherent time averaging is implemented as a primary stage with 20 averaging blocks before using digital filters. Figures 11 to 13 indicate the spectrum results of the output of Butterworth, Chebyshev and Inverse Chebyshev filters respectively (each of order 2) using the proposed method. The obtained results indicate that the digital filters performance is enhanced using this proposed method as compared to the results given in Figs. 8 to 10 .

\section{PROCESSING RESULTS OF MULTIPLE SIGNALS}

This section examines the processing of multiple signals having similar or different power levels. The frequencies of these multiple signals can be chose arbitrarily. In this application, four signals with frequencies $f_{1}=5 \mathrm{kHz}$, $f_{2}=9.5 \mathrm{kHz}, f_{3}=11 \mathrm{kHz}$ and $f_{4}=17.5 \mathrm{kHz}$ are selected. Figure 14 shows the spectrum of the four input signals having similar power level ( $S N R=5 \mathrm{~dB}$ ). It is clear that all signals can be detected above $-15 \mathrm{~dB}$ threshold level.

As the power levels of the multiple input signals and consequently the SNR of each signal become different, the delectability of the weak signals becomes difficult. Figure 15 depicts the spectrun of multiple input signals with different SNRs $\left(S_{N R}=5 \mathrm{~dB}, \mathrm{SNR}_{2}=-10 \mathrm{~dB}, \mathrm{SNR}_{3}=-15 \mathrm{~dB}\right.$ and $\mathrm{SNR}_{4}=0 \mathrm{~dB}$ ). It is shown that the weak signals are completelv immersed in noise. In order to isolate and detect the weak in-band signals, the input signal is applied to the coherent time averaging technique with 20 averaging blocks, and then processed through the digital bandpass filters. 
Figures 16 to 18 illustrate the output of a single stage of fourth order of Butterworth, Chebyshev and Inverse Chebyshev filters respectively using the proposed method. The results show that the Butterworth and Chebyshev filters succeeded in isolating the two weak signals while the Inverse Chebyshev is unable to perform this task due to the high amplitude of the ripples in its stop band.

Now, we are going to examine the use of the proposed method to isolate the weak signals using lower order filters arranged in cascade structure. Figures 19 to 36 illustrate the performance of digital bandpass filters using these cascade structures. Figures 19,22,25 and 31 depict the output of the $1^{\text {st }}$ (second order) stage of Butterworth, Chebyshev $\varepsilon=1$, Inverse Chebyshev $\varepsilon=1$ and Inverse Chebyshev $\varepsilon=0.5$ respectively. The results illustrate that all the second order filters, even with the use of the proposed method, are unable to isolate the weak input signals efficiently. Thus, there is a need for additional stages.

The equivalent transfer function of two similar stages using Butterworth, Chebyshev ( $\varepsilon=1)$, Inverse Chebyshev $(\varepsilon=1)$ and Inverse Chebyshev $(\varepsilon=0.5)$ are given figures $20,23,26$ and 32 respectively. The equivalent transfer function of different stages (Chebyshev and Inverse Chebyshev each with $\varepsilon=1$ ) is illustrated in Fig. 34 . Figure 23 shows that the ripple width in passband increases using cascades of similar Chebyshev filter stages. This results in reducing the level of the output weak signals.

Figures 21, 24, 27 and 33 illustrate the output results of the second stage using similar stages of Butterworth, Chebyshev $(\varepsilon=1)$, Inverse Chebyshev $(\varepsilon=1)$ and Inverse Chebyshev $(\varepsilon=0.5)$ respectively. Figure 35 shows the output result of the second stage using cascade structure of Chebyshev \& Inverse Chebyshev (each with $\varepsilon=1$ ) and figure 36 shows the output result of the second stage using cascade structure of Inverse Chebyshev \& Chebyshev (each with $\varepsilon=1$ ). It is shown that the obtained results in these figures are similar. So, this combination of two different cascade stages improves both filters performance in detecting the weak signals as compared to the result given in Fig. 27.

The two stages of Inverse Chebyshev filters with $\varepsilon=1$ are insufficient to isolate the weak signals. Now, we will add a third similar stage to the structure. The equivalent transfer function of the three similar stages of Inverse Chebyshev filters with $\varepsilon=1$ is given in Fig.28. Figure 29 illustrates the output spectrum of this three stages structure. It is clear that the three stages structure is unable to isolate the weak signals. This problem can be solved using two stages cascade structure of Inverse Chebyshev filters but with $\varepsilon=0.5$. The transfer function of a single stage of Inverse Chebyshev filter with $\varepsilon=0.5$ is illustrated in Fig. 30 and the equivalent transfer function of the similar two stages structure is given in Fig. 32. The output spectrum of this proposed structure is given in Fig. 33. It is clear that good isolation of the weak signals is achieved using this proposed structure.

\section{CONCLUSIONS}

This paper proposed the use of coherent time averaging technique to enhance the digital filters performance in weak signals detection and isolation. It is shown that all second order filter stages of all filter types with and without 
tr a use of the proposed method are unable to isolate the weak signals. By cascading two similar stages of digital Butterworth and Chebyshev filters, we can isolate the weak signals efficiently. Cascading three similar stages of the Inverse Chebyshev digital filter with high $\varepsilon$ is unable to isolate the weak signals even with the proposed method. Reducing the value of $\varepsilon$ for Inverse Chebyshev digital filters improves the detection of weak signals using two similar stages only. Cascading digital filters of different types (Chebyshev and Inverse Chebyshev) yields better results in weak signals delectability.

\section{REFERENCES}

[1] John G. Proakis, Charles M.Rader, Fuyun Ling and Chrysostomos L.Nikias "Advanced Digital Signal Processing" Copyright 1992 by Macmillan Publishing Company.

[2] "Digital Signal Processing" Edited by N.B.Jones. IEE Control Engineering Series 22, 1987, London.

[3] Dessouky, M.I. " Processing of SARSAT Signals Using Modified Digital Lerner Filter" Fifteenth National Radio Science Conference NRSC, Feb., 1998, Cairo, Egypt.

[4] Dessouky, M.I. and Hadhoud, M.M. "Adaptive Technique For Multiple Signal Averaging" Sixth National Radio Science Conference NRSC, Feb., 1989, Cairo, Egypt.

[5] C.M. Thomas, et al., "Signal averaging by parallel digital filters" , IEEE trans. On Acoustics, Speech, and signal processing, vol. ASSP-30, No. 2 , April 1982, PP.338-346.

[6] Andreas Antoniou "Digital Filters: Analysis And Design" McGraw- Hill Book Company, New York, 1979.

[7] Johnson, David E. "Introduction to Filter Theory" Prentice-Hall, Inc.1976. 


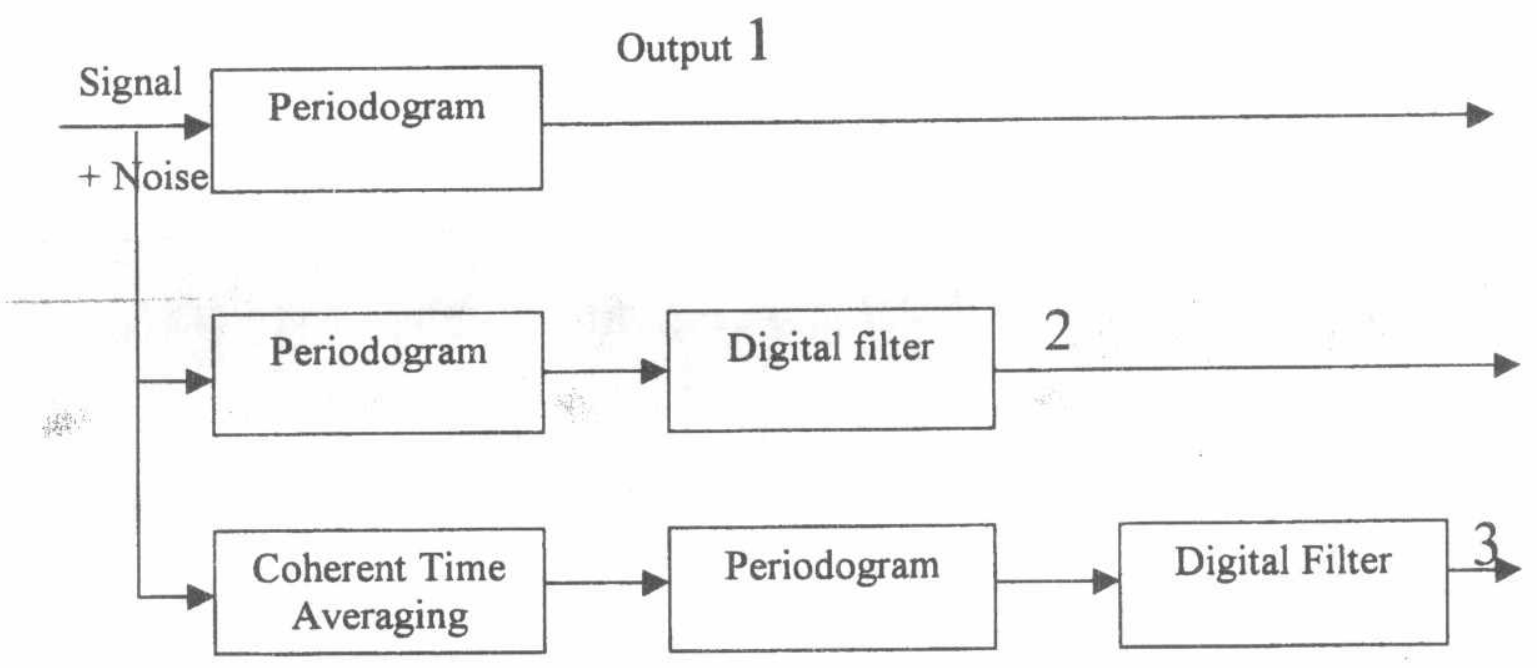

Fig. (I) 


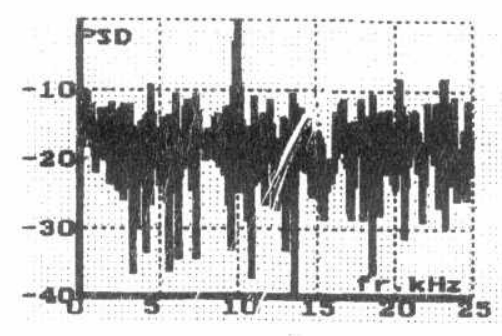

Fig.(2)

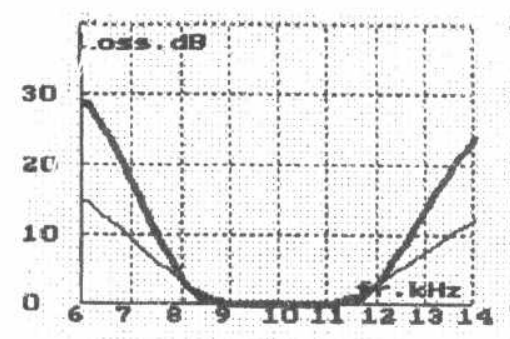

Fig.(5)

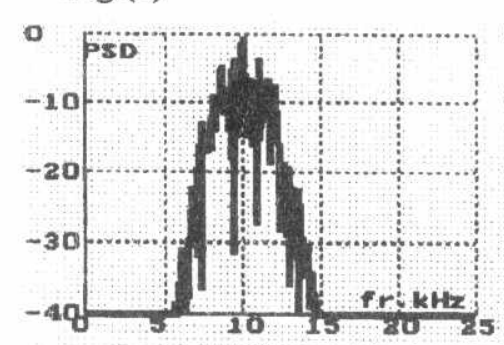

Fig.(8)

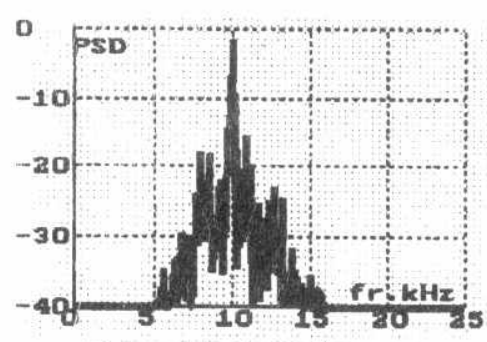

Fig.(11)

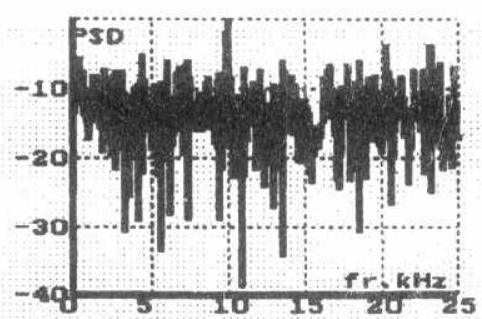

Fig.(3)

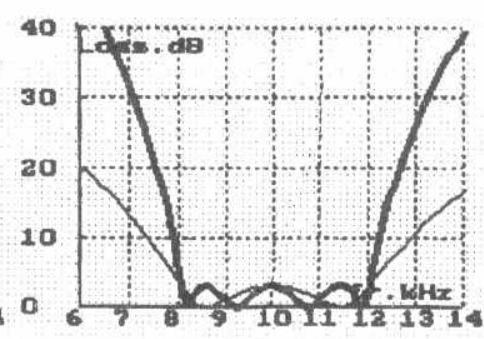

Fig.(6)

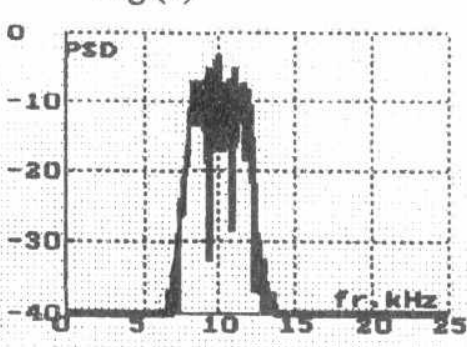

Fig.(9)

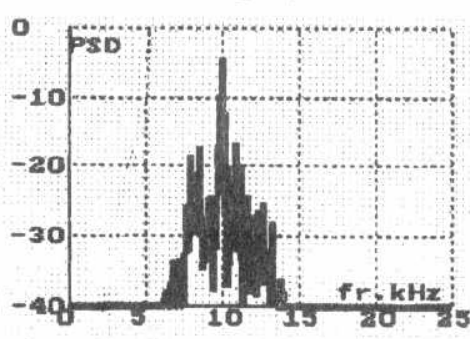

Fig.(12)

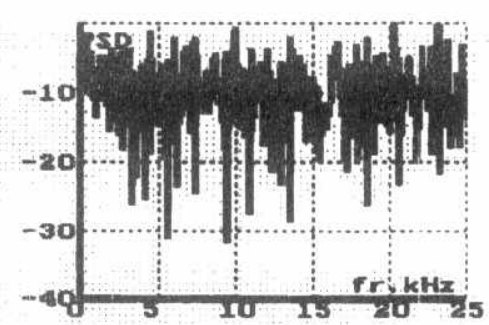

Fig.(4)

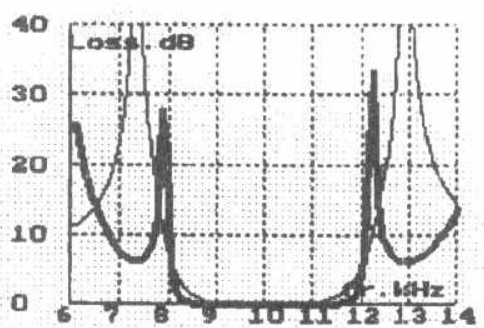

Fig.(7)

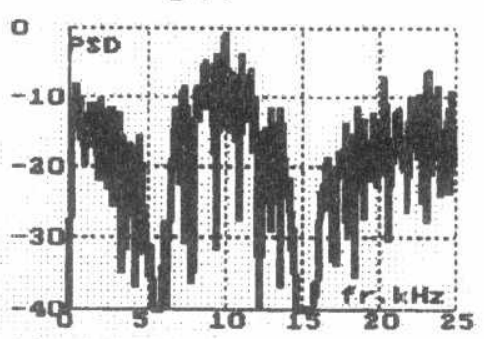

Fig.(10)

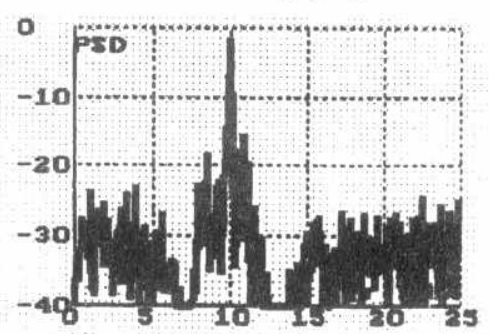

Fig.(13) 


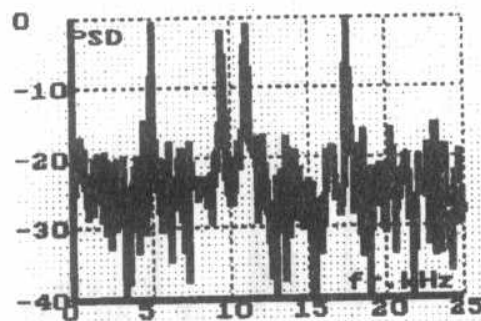

Fig.(14)

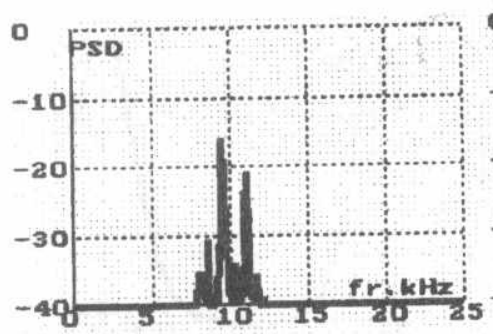

Fig.(16)

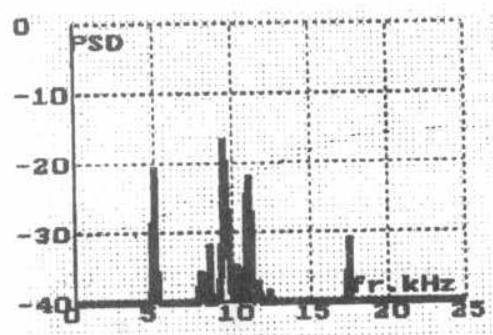

Fig.(19)

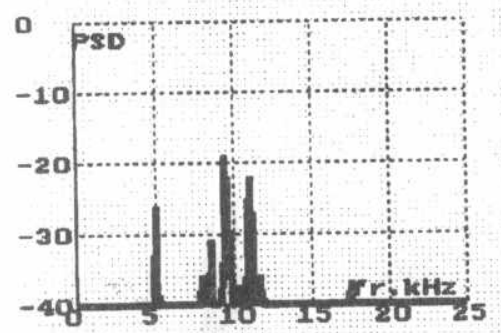

Fig.(22)

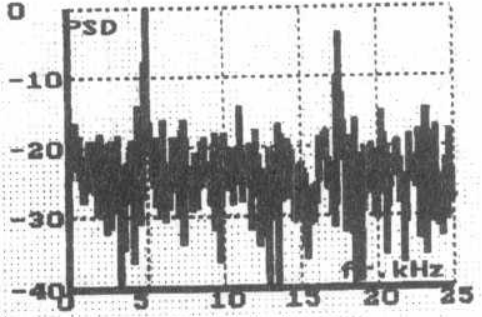

Fig.(15)

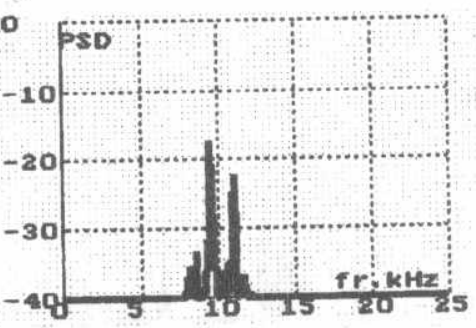

Fig.(17)

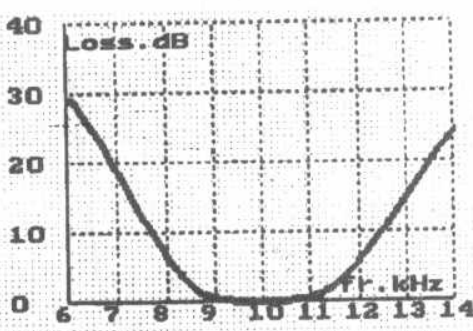

Fig.(20)

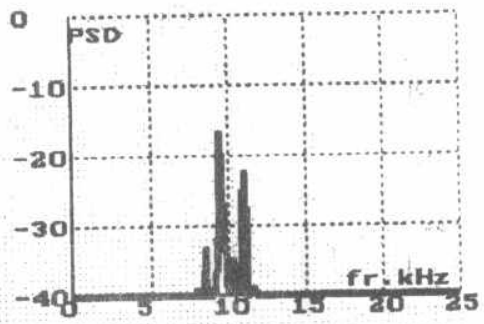

Fig.(21)
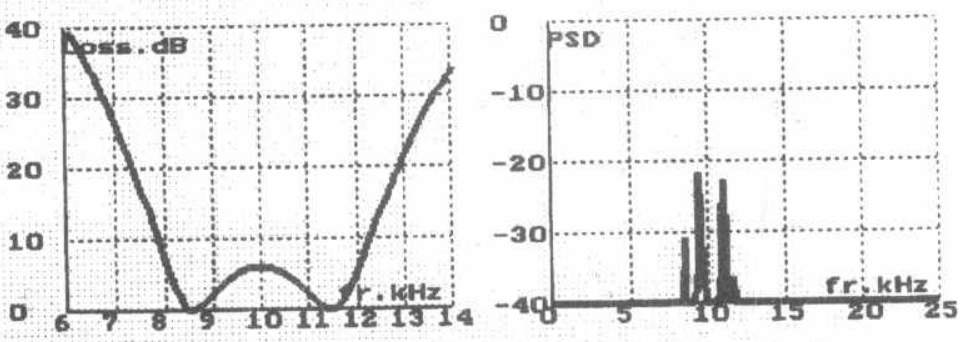

Fig.(24) 


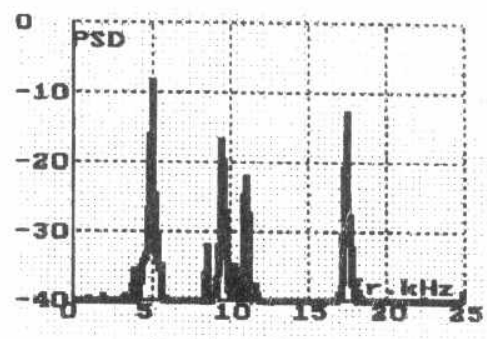

Fig.(25)

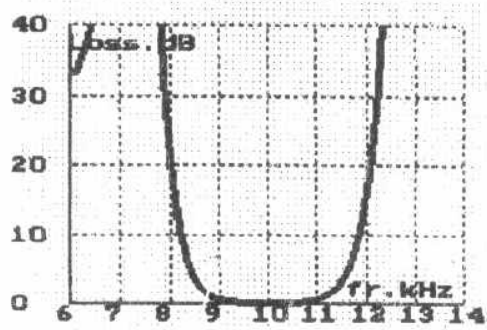

Fig.(28)

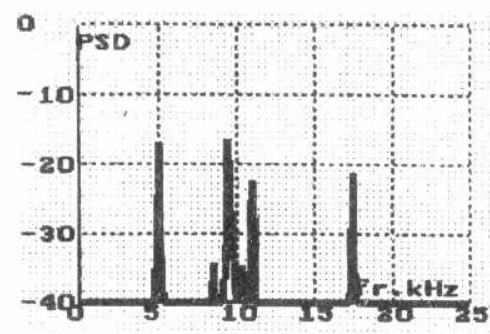

Fig.(31)

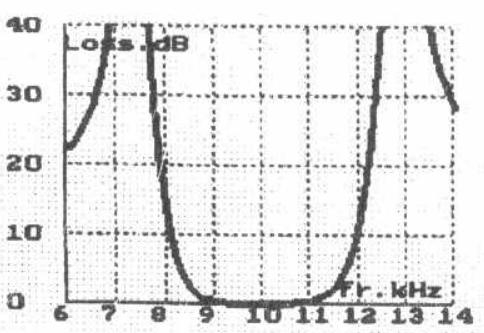

Fig.(26)

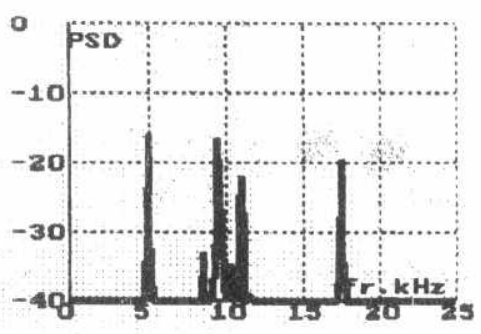

Fig.(27)
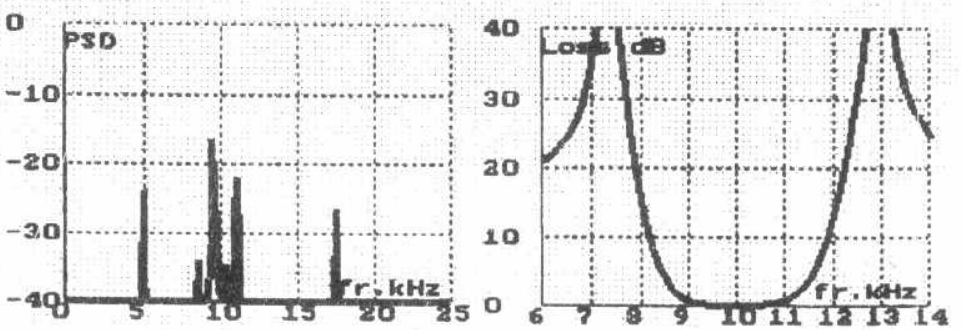

Fig.(29)

Fig.(30)
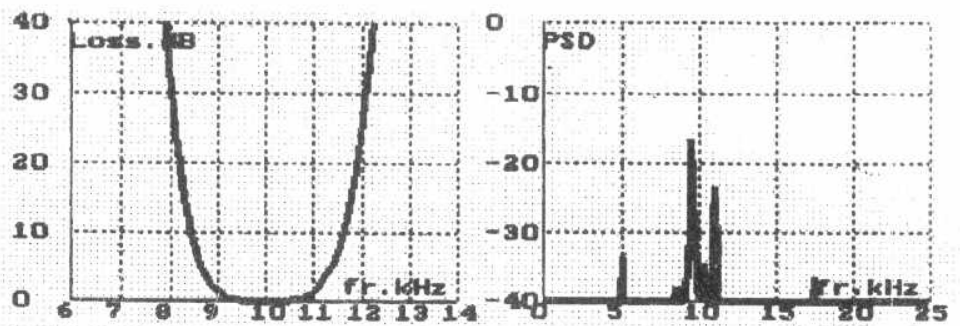

Fig.(33)
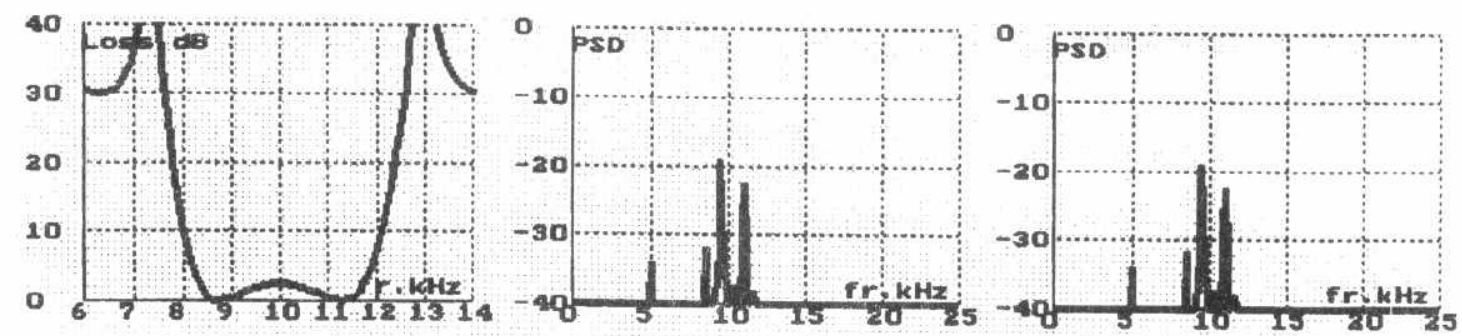

Fig.(34)

Fig.(35)

Fig.(36) 